\title{
A Standardized Protocol for Maximum Repetition Rate Assessment in Children
}

\author{
Sanne Diepeveen ${ }^{a, b}$ Leenke van Haaften ${ }^{b}$ Hayo Terband ${ }^{c}$ Bert de Swart ${ }^{a, b}$ \\ Ben Maassen $^{d}$ \\ ${ }^{a}$ HAN University of Applied Sciences, Nijmegen, The Netherlands; ${ }^{b}$ Donders Institute for Brain, Cognition and \\ Behaviour, Department of Rehabilitation, Radboud University Medical Center, Nijmegen, The Netherlands; ${ }^{C}$ Utrecht \\ Institute of Linguistics-OTS, Utrecht University, Utrecht, The Netherlands; ${ }^{d}$ Centre for Language and Cognition and \\ Research School of Behavioural and Cognitive Neurosciences, University of Groningen, Groningen, The Netherlands
}

\section{Keywords}

Maximum repetition rate $\cdot$ Assessment $\cdot$ Motor speech .

Speech

\begin{abstract}
Background/Aims: Maximum repetition rate (MRR) is often used in the assessment of speech motor performance in older children and adults. The present study aimed to evaluate a standardized protocol for MRR assessment in young children in Dutch. Methods: The sample included 1,524 children of 2-7 years old with no hearing difficulties and Dutch spoken in their nursery or primary school and was representative for children in the Netherlands. The MRR protocol featured mono-, tri-, and bisyllabic sequences and was computer-implemented to maximize standardization. Results: Less than $50 \%$ of the 2 -year-olds could produce $>1$ monosyllabic sequence correctly. Children who could not correctly produce $\geq 2$ monosyllabic sequences could not produce any of the multisyllabic sequences. The effect of instruction ("faster" and "as fast as possible") was small, and multiple attempts yielded a faster MRR in only $20 \%$ of the cases. MRRs did not show clinically relevant differences when calculated over different numbers of repeated syllables. Conclusions: The MRR protocol is suitable for children of 3 years and older. If children cannot produce at least 2 of the monosyllabic sequenc-
\end{abstract}

\begin{tabular}{ll}
\hline KARGER & ( 2019 The Author(s) \\
Published by S. Karger AG, Basel & Karger \\
E-Mail karger@karger.com & This article is licensed under the Creative Commons Attribution- \\
www.karger.com/fpl & $\begin{array}{l}\text { NonCommercial-NoDerivatives 4.0 International License (CC BY- } \\
\text { NC-ND) (http://www.karger.com/Services/OpenAccessLicense). } \\
\text { Usage and distribution for commercial purposes as well as any dis- } \\
\text { tribution of modified material requires written permission. }\end{array}$
\end{tabular}

es, the multisyllabic tasks should be omitted. Furthermore, all fast attempts of each sequence should be analyzed to determine the fastest MRR.

(c) 2019 The Author(s)

Published by S. Karger AG, Basel

\section{Introduction}

Diagnosing a child with motor speech disorders (MSD; e.g., childhood apraxia of speech [CAS], developmental dysarthria) is not always an easy task for a speech-language pathologist (SLP). Children with MSD form a heterogeneous group not only due to etiological factors but also due to individual differences in the pattern of development of the speech disorder leading to individual differences in speech errors [1]. Because of these differences, it is difficult to assess the underlying deficits (issues in speech motor planning, programming, or execution) in children with MSD. In clinical practice, underlying deficits are often examined separately, but multiple factors may be involved [2]. This is why the precise identification of MSD in many children is difficult, especially for those who do not have an evident primary problem [3]. Nevertheless, the precise identification of MSD is important for a correct diagnosis and treatment planning. If the intervention is better adapted to the individual diagnosis, this will ensure better prog-

Sanne Diepeveen

Institute of Health Studies, Speech and Language Therapy

HAN University of Applied sciences, Postbus 6960

NL-6503 GL Nijmegen (The Netherlands)

E-Mail Sanne.diepeveen@ @ han.nl 
ress [3], which can lead to great benefits for further speech and language development of the child.

A misdiagnosis or under-diagnosis can occur because SLPs simply rely on a diagnostic checklist for identifying MSD. These lists are often not specific enough to distinguish between the possible different underlying deficits. At the moment, there is no specific test protocol for diagnosing children with MSD [4]. Recently, Shriberg et al. [5], proposed to use a Pause marker to identify children with CAS. This could be helpful, but there is a need for a gold standard for all children with MSD. Furthermore, there are only a few objective instruments for mapping children's motor speech skills and there is no norm referenced assessment based on a large data set. This is a major problem, as a good understanding of normal speech is necessary for the interpretation of MSDs [6]. Finally, the existing assessments may be hard for children to complete and it also demands a lot of the SLP's judgment ability. The outcomes therefore may not represent the children's true abilities [7].

However, an often-used objective assessment for the clinical judgment of the motor speech performance of older children and adults is the maximum repetition rate (MRR) [8-11]. The MRR frequently contains 2 types of stimuli: repetition of monosyllables (papapa) and of multiple syllable sequences (pataka) [9]. MRR is also called diadochokinetic (DDK), and both terms are used in the literature. We choose the term MRR instead of DDK.

There is much debate about using meaningful (e.g., "patticake" or "pat-a-cake") or nonmeaningful stimuli (e.g., "pataka"); however, Williams and Stackhouse [12] concluded that it is desirable to use nonmeaningful stimuli to assure that the children's performance is not influenced by their linguistic abilities. Furthermore, the MRR contains often the consonants $/ \mathrm{p} /, / \mathrm{t} /$, and $/ \mathrm{k} /$ in a sequence [13] such that the 3 major articular organs are examined, namely, the lips, the jaw, and the tongue [14]. Thus, the different consonants represent multiple levels of physiological complexity since each consonant has a different place of articulation and age of acquisition. These consonants cannot be produced in isolation in succession, which is why the consonants are combined with a vowel [13]. Thus, the syllables $/ \mathrm{pa} /, / \mathrm{ta} /$, and $/ \mathrm{ka} /$ were used in several studies $[3,8,14]$. MRR protocols typically consist of multiple components, which increase in complexity. First, the child should repeat the monosyllabic sequences /papa../, / tata../, and /kaka../. Second, bisyllabic sequences such as / pata../ and /taka../ are administered. The MRR ends with the repetition of the trisyllabic sequences /pataka../ [14]. During the assessment of MRR, children are asked to re- peat the different sequences in one breath at the highest possible pace. The sequences are meant to be repeated without errors and interruptions [14]. Many children struggle with the unnatural situation of the MRR, which requires a specific approach with regard to instruction and practice opportunities [15]. The MRR appears to be difficult for younger children, who make relatively more articulation errors during MRR tasks as compared to conversational speech [16]. Williams and Stackhouse [12] found in their study of 30 typically developing children aged 3-5 that the MRR was more sensitive when the score was based on accuracy and consistency instead of rate of the productions. However, Yaruss and Logan [16] found no age-related increase in such MRR accuracy and consistency scores. Overall, young children show much more variability in their performance than older children, such that the timing, speed, and fluency of speech movements become less variable when children get older [17]. Several studies showed that children make fewer mistakes during the performance of monosyllabic sequences compared to the multisyllabic sequences [18], and the rate of the sequences decreases as the task becomes more complex $[19,20]$.

\section{Measuring $M R R$}

The MRR used to be measured perceptually without any support of instrumental methods that can visualize the acoustic waveform. However, Gadesmann and Miller [21] noted that the use of only perceptual evaluation is not acceptable for clinical diagnosis because perceptual measurement is not accurate enough. Nowadays, there are several programs that semi-automatically interpret the various MRR results. Some examples of these types of programs are the DDK Rate Analysis, which is part of the Motor Speech Profile Model [22], TOCS+ MPT Recorder ${ }^{\mathrm{TM}}$ [23], and Praat [24]. Although in these programs, the task of the examiner is reduced to simply counting syllables, difficulties still occur when the speaker repeats the syllables quickly and irregularly. In this case, the individual syllables are too close together, which makes it difficult to detect the syllable boundaries which will influence the reliability of the value.

There is no uniform method of measuring the MRR, which makes it difficult to compare the results of different children worldwide. There are 3 methods being used: (1) counting syllables repeated in a certain amount of time (count by time), (2) measuring the time needed to repeat a given number of syllables (time by count), or (3) assess how many syllables can be produced in one breath [21]. As a consequence, there is large variability with respect to the collected norm data, which in its turn leads to difficulties with the interpretation of the MRR results $[7,12]$. 
Clinical Use of the MRR

The MRR performance of children with MSD differs compared to typically developing children. Authors [14, $15,25,26]$ of 4 separate studies concluded that children with MSD (spastic dysarthria and CAS) differ in their performance on the MRR. More recently, Murray et al. [27] advised to use an oral motor assessment to diagnose CAS, which includes the trisyllabic sequence, /pataka/, and polysyllabic word accuracy to diagnose CAS. The mentioned authors concluded that the MRR is a valuable tool in the differential diagnosis of underlying speech motor deficits, which is supported by the differences in MRR performances between children diagnosed with dysarthria [15] and apraxia of speech [14] as compared to controls. Others dispute this because they did not find such differences between children with a typical development and children with MSD [28, 29]. Our opinion is that, although MRR does not necessarily reflect the primary speech disorder in all cases with SSD, MRR can play a role in the differential diagnosis to assess disorders in underlying articulomotor planning and programming $[3,30]$. Interpreting only performance on the MRR task is insufficient to assess the underlying speech problem; this requires multiple tasks and the assessment of a comprehensive speech profile [30]. Thus, in a large validation study, we assessed performances on the MRR as well as other speech tasks (picture naming [PN], nonword imitation [NWI], word and nonword repetition [WR, NWR]) with the recently developed diagnostic instrument, Computer Articulation Instrument (CAI) [31] - in a group of 1,524 typically developing children. Factor analyses on the task performances showed separate factors for each of the 4 tasks [32]. The diagnostic value of these norm data resides in now being able to compare MRR performance of children with MSD to typical development.

As discussed above, SLPs use the MRR as an assessment tool for children with MSD. To date, available norm data are based on small samples of children, especially in the younger age groups ( $2-5$ years old), and there is still much debate on the manner to conduct the MRR and the method to calculate the MRR. The aim of this study was to optimize a standardized protocol - which was based on previous studies in the Dutch language $[14,15,25]$. Other research questions of this study were: are children aged 2-7 years able to perform the MRR task, and what kind of instruction and how many attempts do children need to produce their fastest sequence; during clinical work, we noticed some children were slower after the instruction to go as fast as possible.

\section{Materials and Method}

\section{Participants}

This study was part of a large normative study of a new Dutch instrument, the CAI, to assess children's speech problems (for more details see Maassen et al. [31]). A total of 1524 Dutch-speaking children in the Netherlands were recruited between January 2008 and April 2015. The children were recruited via nurseries (47) and mainstream primary schools (71) in the Netherlands. The sample was representative for gender, urbanization, and geographic region. Inclusion criteria were as follows: (1) no hearing difficulties and (2) the Dutch language was spoken in the nursery or primary school. Table 1 shows the number of subjects per MRR sequence per age group (14 age groups were selected) and gender of the children. Not all children executed all MRR tasks. Furthermore, in some cases, the audio files were damaged due to technical problems or the individual syllables were not recognizable because of background noise and the recordings were excluded from the sample.

The children were randomly selected from a list based on age group and gender. All parents/caregivers of the randomly selected children were asked for permission via an informed consent letter to include their child anonymously in this large study. If parents gave permission, they filled out a short questionnaire containing questions about the speech and language development, multilingualism, and health condition (e.g., loss of hearing) of the child. The protocol has been assessed by an ethics committee (Radboud University Nijmegen Medical Centre) and the study was carried out.

\section{Data Collection}

The children were seen individually by 2 SLP students or 1 SLP. In total, 14 SLPs assessed the younger children (2-4 years of age) and 110 SLP students administered the CAI for the older children (4-7 years of age). All these research assistants were trained to assess children with the CAI by the first 2 authors, and a precise instruction in the form of a guideline was given. To assure a flawless administration of the CAI, students worked in pairs.

An assessment session with a child contained the 4 tasks of the CAI: PN, NWI, WR, NWR, and MRR $[31,32]$.

The assessment was administered at the child's nursery or primary school in a quiet room (or the room with the least possible background noise). The CAI was administered using a laptop, and the acoustic signal was automatically stored on the computer's hard disk in one recording for each of the different tasks. The child and research assistant were seated in front of the computer next to each other with a microphone and both wore a headset, or speakers were present, to provide a good sound level of the automated instruction of the CAI. The whole CAI would take approximately 30 min with the MRR being the last section of the CAI. The administration of the MRR took about 5-10 min per child.

\section{MRR Administration}

A protocol (Table 2) for the assessment of the MRR task was developed based on previous studies in Dutch and other languages $[14,15,33]$. The instruction was given by the CAI computer program to maximize standardization, and the children were asked to imitate the following sequences: first 3 monosyllabic sequences (/papa../, /tata../, and /kaka../), followed by 1 trisyllabic sequence (/pataka.../) and finally 2 bisyllabic sequences (/pata../ and /taka../). First, the children were asked to repeat a short sequence of 3 syllables (e.g., /papapa/) in a normal speaking rate, 
Table 1. Age and gender for the 14 age groups of the normative sample

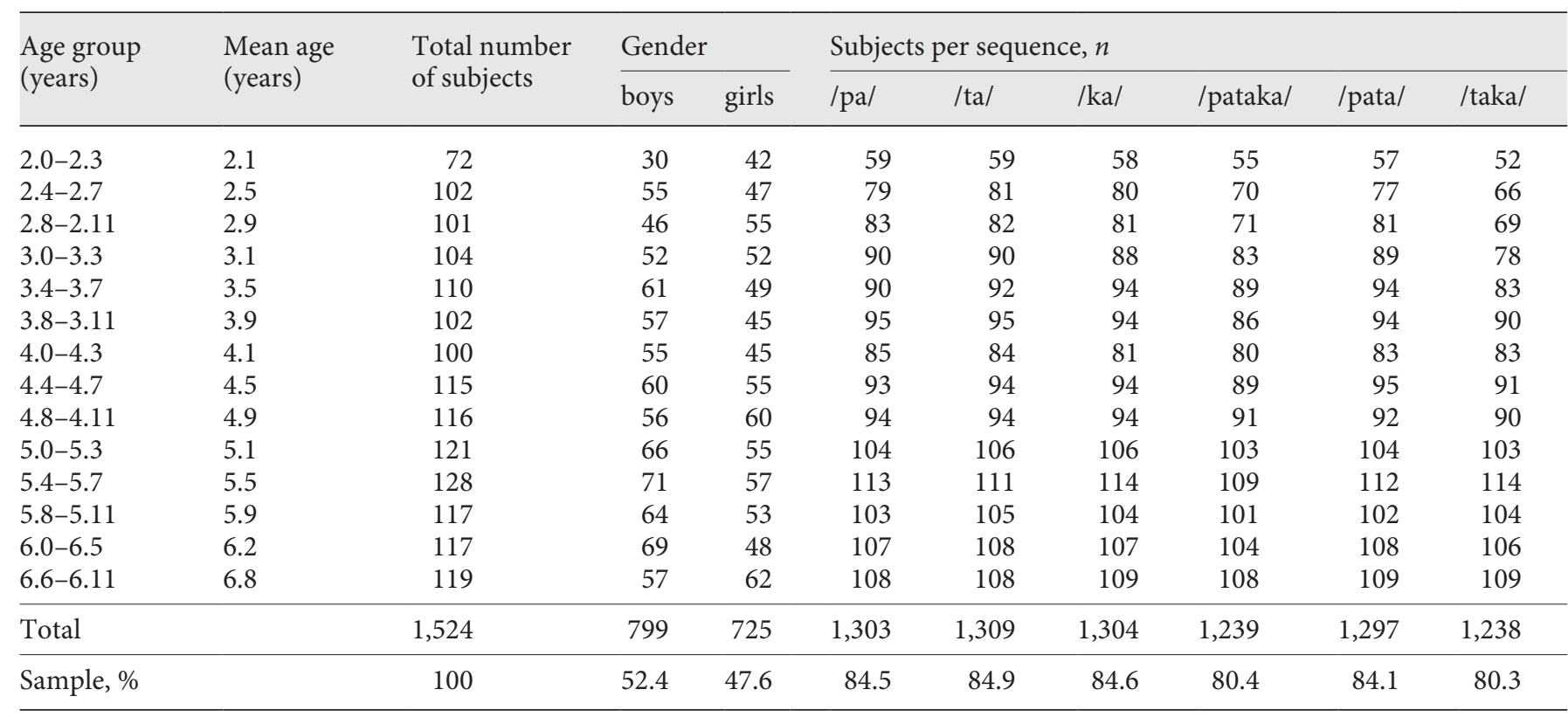

Table 2. Assessment protocol for the MRR

\begin{tabular}{|c|c|c|c|}
\hline & Sequence & Trial & Instruction \\
\hline 1 & $\mathrm{pa}$ & 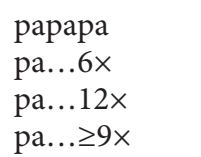 & $\begin{array}{l}\text { Sequence of } 3 \text { syllables, normal speech rate } \\
\text { Sequence of } 6 \text { syllables, normal speech rate } \\
\text { After an audio example, a faster speech rate ( } 5 \text { syllables per second), sequence of } 12 \text { syllables } \\
\text { As fast as possible (without an example) a sequence of minimal } 9 \text { syllables }\end{array}$ \\
\hline 2 & ta & $\begin{array}{l}\text { Tatata } \\
\text { ta...6x } \\
\text { ta...12x } \\
\text { ta... } \geq 9 \times\end{array}$ & $\begin{array}{l}\text { Sequence of } 3 \text { syllables, normal speech rate } \\
\text { Sequence of } 6 \text { syllables, normal speech rate } \\
\text { After an audio example, a faster speech rate ( } 5 \text { syllables per second), sequence of } 12 \text { syllables } \\
\text { As fast as possible (without an example) a sequence of minimal } 9 \text { syllables }\end{array}$ \\
\hline 3 & $\mathrm{ka}$ & $\begin{array}{l}\text { kakaka } \\
\text { ka...6x } \\
\text { ka...12x } \\
\text { ka... } \geq 9 \times\end{array}$ & $\begin{array}{l}\text { Sequence of } 3 \text { syllables, normal speech rate } \\
\text { Sequence of } 6 \text { syllables, normal speech rate } \\
\text { After an audio example, a faster speech rate ( } 5 \text { syllables per second), sequence of } 12 \text { syllables } \\
\text { As fast as possible (without an example) a sequence of minimal } 9 \text { syllables }\end{array}$ \\
\hline 5 & pata & $\begin{array}{l}\text { pata } \\
\text { pata...3x } \\
\text { pata...6x } \\
\text { pata... } \geq 4 \times\end{array}$ & $\begin{array}{l}\text { Sequence of } 2 \text { syllables, normal speech rate } \\
\text { Sequence of } 6 \text { syllables, normal speech rate } \\
\text { After an audio example, a faster speech rate ( } 5 \text { syllables per second), sequence of } 12 \text { syllables } \\
\text { As fast as possible (without an example) a sequence of minimal } 8 \text { syllables }\end{array}$ \\
\hline 6 & taka & $\begin{array}{l}\operatorname{taka} \\
\operatorname{taka} \ldots 3 \times \\
\operatorname{taka...6\times } \\
\operatorname{taka...\geq 4\times }\end{array}$ & $\begin{array}{l}\text { Sequence of } 2 \text { syllables, normal speech rate } \\
\text { Sequence of } 6 \text { syllables, normal speech rate } \\
\text { After an audio example, a faster speech rate ( } 5 \text { syllables per second), sequence of } 12 \text { syllables } \\
\text { As fast as possible (without an example) a sequence of minimal } 8 \text { syllables }\end{array}$ \\
\hline
\end{tabular}

MRR, maximum repetition rate. 
Table 3. Analysis protocol for calculating the MRR

The sequence is considered correct if:

- The syllables are pronounced fluently in succession; dialect variances are accepted

The sequence is considered partially correct if:

- The sequence contains a single error (e.g., /papatapapapa/); then the sequences before and after the error are considered, and the longest and best sequence (at least 3 syllables) is selected

- The sequence contains noise or other interfering elements, but a good sequence can be analyzed before or after the noise or interfering element; then the longest and best sequence is selected (at least 3 syllables)

- The sequence contains pauses or interruptions; then the series are evaluated before and after the pause, and the longest and best sequence (at least 3 syllables) is selected; pauses can arise from:

Inhalation: The child inhales during the execution of the sequence and then continues with the sequence; this also applies to syllables that are pronounced on an inhalation

Rhythm: The child deviates from the rhythm of the sequence and a pause occurs; this is seen in waveform representation with a striking distance between 2 syllables

The sequence is considered incorrect if:

- The sequence in total consists of 4 syllables or less

- The sequence is influenced by phonological processes (e.g., substitution, reduction, assimilation, metathesis, addition); these sequences were marked in an excel file for error analysis

- The sequence is influenced by one of the following issues:

Noise or other interfering elements

- Noise due to an interruption on the part of the examiner or other audible sounds, that makes the individual syllables unrecognizable

Sound volume

- Low volume that makes the individual syllables unrecognizable

Syllables cannot be distinguished

- Syllables in the waveform cannot clearly be distinguished from each other

MRR, maximum repetition rate.

followed by a longer sequence of 6 syllables in a normal rate (e.g., /papapapapapa/). The next instruction included imitation of a series of several syllables at a faster rate (the audio example contained 12 syllables at a faster rate). Finally, the children were asked to produce the syllable sequences as fast as possible. If necessary, the child got 3 attempts for every sequence (the CAI was programmed a maximum of 3 attempts per sequence) to collect an accurate or faster repetition of the sequence; the third attempt was given if the first 2 were both incorrect or the research assistant had the impression that the child could produce a faster rate.

If a child refused to utter a sequence, the research assistant tried to motivate the child and the sequence would be repeated or the research assistant presented the next sequence. If the child kept on refusing during the next sequences, the session was ended.

\section{MRR Analysis}

After all the data with the basic protocol were collected, the process of analyzing the samples started with the goal to maybe alter the protocol procedure of assessing and analyzing the MRR task. Since the program stored one whole recording of all trials per child, the recordings were cut in smaller sequences by hand with Praat software, version 6.0.21 [24].

Six students of HAN University of Applied Sciences and 3 SLPs analyzed the mono-, tri-, and bisyllable sequences according to a protocol, which is shown in Table 3 . They were all trained by the first author and started with analyzing one practice sample of one audio-recording, which contained all the 6 sequences. The stu- dents received instructions on how to use and interpret the protocol (e.g., which syllable sequence is suitable for further analyses if the child took a breath or pause?). Only the last 2 items of the MRR task were analyzed (those elicited by the instructions "faster" and "as fast as possible"). Any occurring speech errors were registered per sequence in an excel file (e.g., /papadada/).

The audio-recordings, containing just one sequence and attempt, were analyzed by the first author and one of the SLPs with the help of a customized Praat-script (developed by one of the authors; HT). The script detected and marked syllable onsets by localizing the noise burst of the voiceless plosives. The first and the last syllables were excluded because speakers often produce the first syllable with a longer duration and higher intensity [14] and the last syllable is also often lengthened [34]. Before extracting the syllable durations and MRR, the marked syllable onsets were depicted in the waveform and inspected visually, and any errors in the number of syllables indicated by the script were corrected manually. If corrected manually, the script could not give the separate durations of all the individual syllables and only the MRR value (total number of syllables divided by total duration of the sequence) was given. Figure 1 gives an example of one of the sequences with the markers. Only sequences with a remaining minimum of 3 syllables were included in the analysis. In some cases, the script could not detect syllable onsets correctly. These samples were analyzed by hand to determine the number of syllables and the duration of the sequence. Eventually, all data of the MRR were merged in SPSS version 24 for Windows (SPSS Inc., Chicago, IL, USA). 


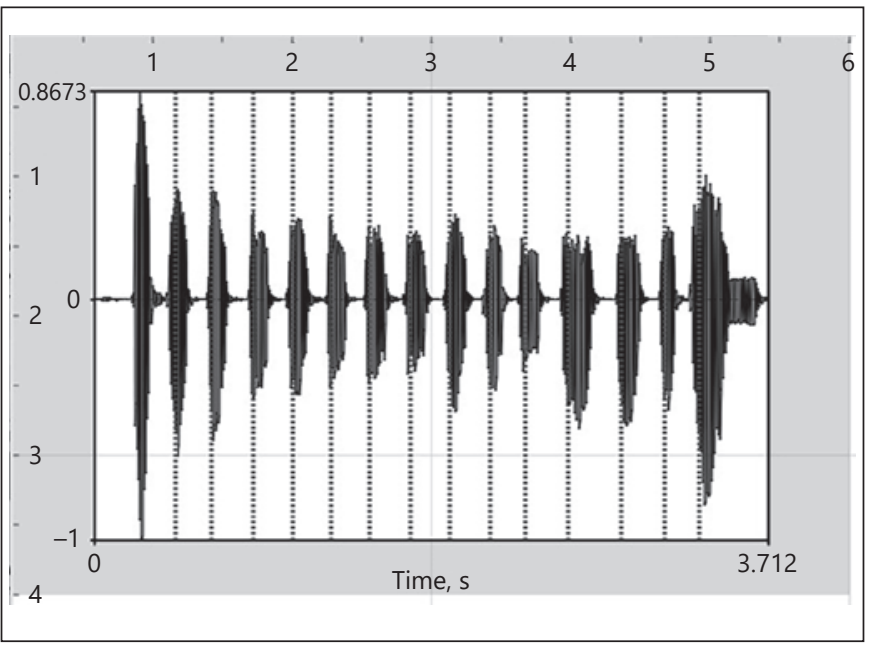

Fig. 1. Example of the analysis with the Praat-script of one sequence /tatata/, fastest first attempt.

\section{Reliability}

Interrater and test - retest reliability were examined and described. We will only describe the result of this study; all details can be found in the publication by van Haaften et al. [32, 35]. Interrater reliability was good for the monosyllabic sequences / pa/ (intraclass correlation coefficient [ICC] 0.81 ) and $/ \mathrm{ka} /$ (ICC 0.83) and sufficient for /ta/ (ICC 0.77). For /pataka/, /pata/, and /taka/, we found insufficient interrater reliability with ICCs ranging from 0.41 to 0.62 . Especially, the younger children had difficulty performing the /pataka/, /pata/, and /taka/; had we included whether the attempts were successful or not, the ICC might have been much higher. Test - retest reliability showed a sufficient reliability measure (ICC 0.70 ) for $/ \mathrm{pa} /$, and for the other sequences, the test - retest reliability was insufficient with ICCs ranging from 0.18 to 0.60 . Reasons for these low scores could be the rapid development of the younger children during the interval between test and retest or a test- retest training effect because children were significantly more competent on /pataka/ on the second test than on the first test $(t[53]=-3.02, p=0.004)$.

\section{Statistical Analysis}

First, frequency tables were constructed to determine how many children produced the different sequences of the MRR. Then, a comparison was made between the completion of the different sequences, for example, monosyllabic sequences versus multisyllabic sequences. Frequency tables were also constructed for the MRR value of the different sequences and attempts. Means and SDs of all parameters were calculated per age group, and repeated-measures analysis of variances was conducted to compare the best performance on the fast (with example) and the fastest (without example) attempt per sequence. Furthermore, to determine if the gold standard of 10 syllables should be maintained, means and SDs over all the first 3-10 syllables each were calculated per sequence and differences were investigated. Furthermore, ICCs with 2-way mixed-effects models featuring no fixed effects were calculated between the MRRs over each of the numbers of syllables compared with the gold standard of 10 syllables.

\section{Results}

First, the results of all children are described to answer the questions if children of all ages can perform the MRR tasks and if all children can perform all the different MRR tasks (mono-, bi-, and trisyllabic sequences). Subsequently, we investigate whether one of the instructions (fast or faster) elicits faster MRRs and whether it matters to ask multiple attempts per sequence. The last part of the results addresses the question if there is a difference in MRR when calculated over $<10$ syllables per sequence.

\section{Ability to Perform the MRR Task}

Tables 4 and 5 show the number of children executing and failing the different sequences of the MRR. For the 2-4-year-olds, not all audio-recordings included all MRR sequences because sometimes the child refused to utter one or multiple sequences and sometimes the SLP foresaw that the child would not execute the bi- and trisyllabic sequences after finishing the monosyllabic sequences. These cases are marked No sequence in Tables 4 and 5. A sequence is marked Fail if the child refused to complete the sequence, if not enough syllables were detected (minimum of 3), if an irregular rhythm (distinct pause) was executed, or if the child made errors (e.g., /pada/ instead of /pata/). For / pa/, 62 children refused to utter any syllables, for /ta/ 91 children, /ka/ 77 children, /pataka/ 156 children, / pata/ 100 children, and /taka/ 129 children. For each of the monosyllabic sequences, the results show that about $80 \%$ of children could produce the sequence correctly. For the multisyllabic sequences, the percentage of children that could produce the sequence correctly is lower, that is, $65.1 \%$ for /pataka/ and slightly higher percentages for /pata/ (75.9) and /taka/ (77.7\%).

Next, we investigated the number of correctly produced sequences per individual. Table 6 provides an overview of the number of monosyllabic sequences that children in the different age groups have performed, showing that only $21 \%$ of the children under the age of 3 can perform all 3 monosyllabic sequences.

In order to determine the capability of carrying out the bi- and trisyllabic sequences in relation to the children's abilities to produce the monosyllabic sequences, we crosstabulated the number of correctly produced monosyllabic sequences with the correct production of the bi- and trisyllabic sequences (Table 7). The results indicate that children who can produce at least 2 monosyllabic sequences are more likely to subsequently also correctly produce a bi- or trisyllabic sequence. The children who can only produce $<2$ monosyllabic sequence have a much 
Table 4. Fail and pass of all monosyllabic sequences

\begin{tabular}{|c|c|c|c|c|c|c|c|c|c|c|c|c|c|c|c|}
\hline \multirow{3}{*}{$\begin{array}{l}\text { Age group } \\
\text { (years) }\end{array}$} & \multicolumn{5}{|l|}{$/ \mathrm{pa} /$} & \multicolumn{5}{|l|}{$/ \mathrm{ta} /$} & \multicolumn{5}{|l|}{ /ka/ } \\
\hline & \multirow[t]{2}{*}{$n$} & \multirow[t]{2}{*}{ pass, $\%$} & \multicolumn{2}{|l|}{ fail, $\%$} & \multirow{2}{*}{$\begin{array}{l}\text { no } \\
\text { sequence, } \%\end{array}$} & \multirow[t]{2}{*}{$n$} & \multirow[t]{2}{*}{ pass, $\%$} & \multicolumn{2}{|l|}{ fail, $\%$} & \multirow{2}{*}{$\begin{array}{l}\text { no } \\
\text { sequence, } \%\end{array}$} & \multirow[t]{2}{*}{$n$} & \multirow[t]{2}{*}{ pass, $\%$} & \multicolumn{2}{|l|}{ fail, $\%$} & \multirow{2}{*}{$\begin{array}{l}\text { no } \\
\text { sequence, } \%\end{array}$} \\
\hline & & & errors & $<3$ syllable & & & & errors & $<3$ syllable & & & & errors & $<3$ syllable & \\
\hline $2.0-2.4$ & 59 & 30.5 & 1.7 & 39.0 & 28.8 & 59 & 32.2 & 1.7 & 32.2 & 33.9 & 58 & 34.5 & 3.4 & 37.9 & 24.1 \\
\hline $2.4-2.8$ & 79 & 35.4 & 2.5 & 44.3 & 17.7 & 81 & 34.6 & 3.7 & 37.0 & 24.7 & 80 & 35.0 & 6.3 & 32.5 & 26.3 \\
\hline $2.8-3.0$ & 83 & 66.3 & 3.6 & 21.7 & 8.4 & 82 & 64.6 & 1.2 & 20.7 & 13.4 & 81 & 63.0 & 3.7 & 23.5 & 9.9 \\
\hline $3.0-3.4$ & 90 & 71.1 & 1.1 & 18.9 & 8.9 & 90 & 75.6 & 0.0 & 13.3 & 11.1 & 88 & 75.0 & 2.3 & 14.8 & 8.0 \\
\hline $3.4-3.8$ & 90 & 66.7 & 5.6 & 20.0 & 7.8 & 92 & 71.7 & 1.1 & 14.1 & 13.0 & 94 & 69.1 & 5.3 & 18.1 & 7.4 \\
\hline $3.8-4.0$ & 95 & 90.5 & 1.1 & 5.3 & 3.2 & 95 & 87.4 & 2.1 & 5.3 & 5.3 & 94 & 88.3 & 3.2 & 3.2 & 5.3 \\
\hline $4.0-4.4$ & 85 & 85.9 & 7.1 & 3.5 & 3.5 & 84 & 90.5 & 2.4 & 3.6 & 3.6 & 81 & 91.4 & 1.2 & 2.5 & 4.9 \\
\hline $4.4-4.8$ & 93 & 96.8 & 0.0 & 1.1 & 2.2 & 94 & 93.6 & 2.1 & 2.1 & 2.1 & 94 & 92.6 & 3.2 & 1.1 & 3.2 \\
\hline $4.8-5.0$ & 94 & 97.9 & 0.0 & 1.1 & 1.1 & 94 & 97.9 & 1.1 & 0.0 & 1.1 & 94 & 96.8 & 0.0 & 0.0 & 3.2 \\
\hline $5.0-5.4$ & 104 & 99.0 & 1.0 & 0.0 & 0.0 & 106 & 94.3 & 0.9 & 1.9 & 2.8 & 106 & 95.3 & 1.9 & 0.0 & 2.8 \\
\hline $5.4-5.8$ & 113 & 99.1 & 0.0 & 0.9 & 0.0 & 111 & 94.6 & 2.7 & 0.0 & 2.7 & 114 & 95.6 & 2.6 & 0.0 & 1.8 \\
\hline $5.8-6.0$ & 103 & 99.0 & 1.0 & 0.0 & 0.0 & 105 & 100.0 & 0.0 & 0.0 & 0.0 & 104 & 99.0 & 1.0 & 0.0 & 0.0 \\
\hline $6.0-6.6$ & 107 & 98.1 & 1.9 & 0.0 & 0.0 & 108 & 99.1 & 0.9 & 0.0 & 0.0 & 107 & 98.1 & 1.9 & 0.0 & 0.0 \\
\hline $6.6-7.0$ & 108 & 99.1 & 0.9 & 0.0 & 0.0 & 108 & 97.2 & 1.9 & 0.0 & 0.9 & 109 & 99.1 & 0.9 & 0.0 & 0.0 \\
\hline Total & 1,303 & 1,095 & 24 & 122 & 62 & 1,309 & 1,095 & 20 & 103 & 91 & 1,304 & 1,091 & 33 & 103 & 77 \\
\hline Sample, \% & 100.0 & 84.0 & 1.84 & 9.4 & 4.8 & 100.0 & 83.7 & 1.5 & 7.9 & 6.9 & 100.0 & 83.7 & 2.5 & 7.9 & 5.9 \\
\hline
\end{tabular}

Table 5. Fail and pass of all tri- and bisyllabic sequences

\begin{tabular}{|c|c|c|c|c|c|c|c|c|c|c|c|c|c|c|c|}
\hline \multirow{3}{*}{$\begin{array}{l}\text { Age group } \\
\text { (years) }\end{array}$} & \multicolumn{5}{|c|}{ /pataka/ } & \multicolumn{5}{|l|}{ /pata/ } & \multicolumn{5}{|l|}{ /taka/ } \\
\hline & \multirow[t]{2}{*}{$n$} & \multirow[t]{2}{*}{ pass, $\%$} & \multicolumn{2}{|l|}{ fail, \% } & \multirow{2}{*}{$\begin{array}{l}\text { no } \\
\text { sequence, } \%\end{array}$} & \multirow[t]{2}{*}{$n$} & \multirow[t]{2}{*}{ pass, $\%$} & \multicolumn{2}{|l|}{ fail, \% } & \multirow{2}{*}{$\begin{array}{l}\text { no } \\
\text { sequence, } \%\end{array}$} & \multirow[t]{2}{*}{$n$} & \multirow[t]{2}{*}{ pass, $\%$} & \multicolumn{2}{|l|}{ fail, $\%$} & \multirow{2}{*}{$\begin{array}{l}\text { no } \\
\text { sequence, \% }\end{array}$} \\
\hline & & & errors & $<3$ syllable & & & & errors & $<3$ syllable & & & & errors & $<3$ syllable & \\
\hline $2.0-2.4$ & 55 & 16.4 & 23.6 & 18.2 & 41.8 & 57 & 22.8 & 15.8 & 35.1 & 26.3 & 52 & 23.1 & 13.5 & 11.5 & 51.9 \\
\hline $2.4-2.8$ & 70 & 17.1 & 24.3 & 20.0 & 38.6 & 77 & 29.9 & 11.7 & 32.5 & 26.0 & 66 & 34.8 & 15.2 & 13.6 & 36.4 \\
\hline $2.8-3.0$ & 71 & 23.9 & 32.4 & 11.3 & 32.4 & 81 & 45.7 & 11.1 & 24.7 & 18.5 & 69 & 52.2 & 10.1 & 10.1 & 27.5 \\
\hline $3.0-3.4$ & 83 & 47.0 & 21.7 & 13.3 & 18.1 & 89 & 57.3 & 14.6 & 13.5 & 14.6 & 78 & 66.7 & 10.3 & 7.7 & 15.4 \\
\hline $3.4-3.8$ & 89 & 42.7 & 24.7 & 9.0 & 23.6 & 94 & 60.6 & 10.6 & 18.1 & 10.6 & 83 & 59.0 & 14.5 & 6.0 & 20.5 \\
\hline $3.8-4.0$ & 86 & 62.8 & 17.4 & 7.0 & 12.8 & 94 & 75.5 & 9.6 & 9.6 & 5.3 & 90 & 86.7 & 4.4 & 1.1 & 7.8 \\
\hline $4.0-4.4$ & 80 & 68.8 & 17.5 & 0.0 & 13.8 & 83 & 80.7 & 9.6 & 2.4 & 7.2 & 83 & 80.7 & 9.6 & 1.2 & 8.4 \\
\hline $4.4-4.8$ & 89 & 75.3 & 18.0 & 1.1 & 5.6 & 95 & 89.5 & 4.2 & 1.1 & 5.3 & 91 & 89.0 & 6.6 & 0.0 & 4.4 \\
\hline $4.8-5.0$ & 91 & 68.1 & 24.2 & 0.0 & 7.7 & 92 & 84.8 & 10.9 & 0.0 & 4.3 & 90 & 81.1 & 13.3 & 0.0 & 5.6 \\
\hline $5.0-5.4$ & 103 & 79.6 & 14.6 & 0.0 & 5.8 & 104 & 91.3 & 4.8 & 1.0 & 2.9 & 103 & 84.5 & 12.6 & 1.0 & 1.9 \\
\hline $5.4-5.8$ & 109 & 83.5 & 12.8 & 0.0 & 3.7 & 112 & 89.3 & 7.1 & 0.0 & 3.6 & 114 & 91.2 & 5.3 & 0.0 & 3.5 \\
\hline $5.8-6.0$ & 101 & 91.1 & 8.9 & 0.0 & 0.0 & 102 & 94.1 & 5.9 & 0.0 & 0.0 & 104 & 91.3 & 7.7 & 0.0 & 1.0 \\
\hline $6.0-6.6$ & 104 & 86.5 & 11.5 & 0.0 & 1.9 & 108 & 95.4 & 4.6 & 0.0 & 0.0 & 106 & 94.3 & 5.7 & 0.0 & 0.0 \\
\hline $6.6-7.0$ & 108 & 91.7 & 7.4 & 0.0 & 0.9 & 109 & 100.0 & 0.0 & 0.0 & 0.0 & 109 & 96.3 & 3.7 & 0.0 & 0.0 \\
\hline Total & 1,239 & 807 & 218 & 58 & 156 & 1,297 & 985 & 105 & 107 & 100 & 1,238 & 962 & 111 & 36 & 129 \\
\hline Sample, \% & 100.0 & 65.1 & 17.6 & 4.7 & 12.6 & 100.0 & 75.9 & 8.1 & 8.3 & 7.7 & 100.0 & 77.7 & 9.0 & 2.9 & 10.4 \\
\hline
\end{tabular}

lower chance of performing a tri- and bisyllabic sequences, showing a weak positive relation ( $\mathrm{rs}=0.278, n=1,524$, $p<0.001)$.

\section{Choosing the Best Sequence and the Number of \\ Syllables}

Children under 35 months of age have more difficulty with executing the different sequences for that reason these children were excluded in further analyses which resulted in the inclusion of 1,041 children.

During data collection, the question raised which attempt or sequence (the one after the instruction "faster" or "as fast as possible") would actually be the fastest MRR, as we observed that children do not always go faster if they have been instructed to go as fast as possible. In addition, some children got up to 3 attempts to produce a sequence 
Table 6. Numbers of children producing $0-3$ of the 3 monosyllabic sequences correctly per age group

\begin{tabular}{lrrrr}
\hline \multirow{2}{*}{$\begin{array}{l}\text { Age group } \\
\text { (years) }\end{array}$} & \multicolumn{5}{c}{ Monsyllabic sequence } \\
\cline { 2 - 5 } & 0 & 1 & 2 & 3 \\
\hline $2.0-2.4$ & 42 & 12 & 9 & 9 \\
$2.4-2.8$ & 58 & 18 & 12 & 14 \\
$2.8-3.0$ & 32 & 14 & 20 & 35 \\
$3.0-3.4$ & 23 & 13 & 19 & 49 \\
$3.4-3.8$ & 31 & 14 & 18 & 47 \\
$3.8-4.0$ & 8 & 8 & 14 & 72 \\
$4.0-4.4$ & 18 & 5 & 13 & 64 \\
$4.4-4.8$ & 23 & 2 & 8 & 82 \\
$4.8-5.0$ & 23 & 0 & 4 & 89 \\
$5.0-5.4$ & 16 & 2 & 7 & 96 \\
$5.4-5.8$ & 14 & 3 & 10 & 101 \\
$5.8-6.0$ & 12 & 1 & 3 & 101 \\
$6.0-6.6$ & 9 & 0 & 7 & 101 \\
$6.6-7.0$ & 10 & 0 & 7 & 102 \\
\hline Total & 319 & 92 & 151 & 962 \\
\hline Sample, $\%$ & 20.9 & 6.0 & 9.9 & 63.1 \\
\hline
\end{tabular}

fast or as fast as possible because the administrator estimated that the child could go faster. As there is no evidence in literature, to our knowledge, regarding which attempt is the fastest overall, we compared the performance of the children after the instructions "fast" and "as fast as possible," as well as the performances on the extra attempts. Of the total group, 742 children got $>1$ attempt for at least one of the sequences. To determine whether instruction has an effect on the realized rate, we compared the best attempt of the children on the "fast" instruction with the best attempts on the "as fast as possible" instruction (Table 8). A repeated-measures analysis of variance yielded significant effects of instruction for all sequences except for $/ \mathrm{ka} /$ (Table 8 ), indicating that for all sequences except $/ \mathrm{ka} /$, children achieved a higher rate on average upon the instruction "as fast as possible" as compared to the preceding "faster" instruction. However, the data must be interpreted with caution, since the effect sizes are rather small, in particular for the monosyllabic sequences (Table 8).

\section{Number of Syllables}

In recent MRR protocols [9, 14-16], the number of syllables that are required/prescribed for analysis is 10 or 12 syllables per sequence. Our clinical experience, however, is that not many children can produce 10- or 12-syllable sequences, especially children with MSD. Because the aim of our research project is to develop an assessment for children with MSD, it is important to evaluate if the protocol can also be administered with $<10$ syllables. Therefore, Kruskal-Wallis test (none of the sequences met the test for equality of variance) per sequence was executed to see if there are differences between the MRR values for each of the sequence lengths with a minimum of 3 syllables and combining the sequences longer than 10 syllables. Sequence /pa/ showed a significant result, and no significant difference in syllable rate between the different sequence lengths was observed for the other sequences (Table 9). In Table 10, the descriptive values of the mono-, tri-, and bisyllabic sequences are presented.

To compare MRR when calculated over different numbers of syllables per child and not between the children as described in Table 10, the MRRs of each successive number of syllables were calculated for children who produced 10 or more syllables in a sequence. Differences between the mean syllable rate for each of the successive sequence lengths from 3 to 9 were studied by estimating ICCs. Table 11 shows good to excellent ICCs for every sequence length (except for the mean syllable rate of sequence length 3 in comparison with the mean rate sequence length 10 of /pata/, which has a moderate ICC).

\section{Discussion}

In this study, we adapted an existing MRR protocol and evaluated this protocol in a sample of 1,524 typically developing Dutch children from 2 to 7 years old; the largest group of children of which MRR assessment is described thus far. The results showed first that children under 30 months of age have severe difficulty with executing the tasks properly and even for children up to 3 years of age, it is still difficult. Most of the previous studies $[16,36]$ described groups of children from 3 years and older, simply because this is the youngest age at which children tend to be referred to an SLP [37]. Although there still is much debate about administering the MRR at this young age, these studies concluded that children from 3 years of age can perform the MRR task. The present results corroborate and extend these findings in a large sample, showing that administering MRR tasks in younger children is indeed problematic. For that reason, we conclude that MRR should not be assessed in children under the age of 3 and we adjusted the MRR protocol for future use accordingly (which is part of the CAI test battery).

Second, the results showed that children who have difficulty performing the monosyllabic series cannot per- 
Table 7. Cross-tabulation of numbers of children correctly producing 0-3 bi- or trisyllabic sequences in relation to their number of correctly produced monosyllabic sequences

\begin{tabular}{|c|c|c|c|c|c|c|c|c|c|c|}
\hline \multirow{2}{*}{$\begin{array}{l}\text { Monosyllabic } \\
\text { sequences }\end{array}$} & \multicolumn{10}{|c|}{ Tri- and bisyllabic sequences } \\
\hline & $n$ & $\%$ & $n$ & $\%$ & $n$ & $\%$ & $n$ & $\%$ & $n$ & $\%$ \\
\hline None & 300 & 19.7 & 15 & 1.0 & 3 & 0.2 & 1 & 0.1 & 319 & 20.9 \\
\hline One & 50 & 5.3 & 17 & 1.1 & 17 & 1.1 & 8 & 0.7 & 92 & 6.0 \\
\hline Two & 29 & 1.9 & 24 & 1.6 & 56 & 3.7 & 42 & 3.8 & 151 & 9.9 \\
\hline Total & 412 & 27.0 & 140 & 9.2 & 302 & 19.8 & 670 & 44.0 & 1,524 & 100.0 \\
\hline
\end{tabular}

Table 8. Comparison of the best performance on the 2 instructions "faster" (with example) and "as fast as possible" (without example)

\begin{tabular}{|c|c|c|c|c|c|c|c|c|}
\hline Sequence & Instruction & $n$ & MRR mean & $\mathrm{SD}$ & df & $\mathrm{F}$ & $p$ value & $\omega^{2}$ \\
\hline \multirow[t]{2}{*}{$/ \mathrm{pa} /$} & Faster & 790 & 4.4 & 0.6 & \multirow[t]{2}{*}{1,550} & \multirow[t]{2}{*}{26.601} & \multirow[t]{2}{*}{$<0.001$} & \multirow[t]{2}{*}{0.009} \\
\hline & Fastest & 752 & 4.5 & 0.7 & & & & \\
\hline \multirow[t]{2}{*}{$/ \mathrm{ta} /$} & Faster & 821 & 4.2 & 0.5 & \multirow[t]{2}{*}{1,615} & \multirow[t]{2}{*}{56.115} & \multirow[t]{2}{*}{$<0.001$} & \multirow[t]{2}{*}{0.0019} \\
\hline & Fastest & 719 & 4.4 & 0.7 & & & & \\
\hline \multirow[t]{2}{*}{ /pataka/ } & Faster & 687 & 3.7 & 0.7 & \multirow[t]{2}{*}{1,411} & \multirow[t]{2}{*}{84.558} & \multirow[t]{2}{*}{$<0.001$} & \multirow[t]{2}{*}{0.049} \\
\hline & Fastest & 627 & 4.1 & 1.0 & & & & \\
\hline \multirow[t]{2}{*}{ /pata/ } & Faster & 735 & 4.1 & 0.6 & \multirow[t]{2}{*}{1,477} & \multirow[t]{2}{*}{88.687} & \multirow[t]{2}{*}{$<0.001$} & \multirow[t]{2}{*}{0.049} \\
\hline & Fastest & 707 & 4.5 & 0.9 & & & & \\
\hline
\end{tabular}

$n$ gives the number of children who produced the sequence; the statistical test and the calculation of mean and SD were conducted with repeated-measures ANOVA on less $(\mathrm{df}+1)$ pairwise comparisons.

MRR, maximum repetition rate.

Table 9. Kruskal-Wallis statistics for the comparison of MRR values for the different numbers of syllables in the produced sequences

\begin{tabular}{lllll}
\hline Sequence & $n$ & df & H & $p$ value \\
\hline /pa/ & 983 & 8 & 20.29 & 0.009 \\
/ta/ & 987 & 8 & 12.99 & 0.112 \\
$/$ ka/ & 981 & 8 & 14.21 & 0.076 \\
/pataka/ & 893 & 8 & 7.472 & 0.487 \\
/pata/ & 953 & 8 & 13.82 & 0.087 \\
/taka/ & 934 & 8 & 15.51 & 0.050
\end{tabular}

MRR, maximum repetition rate. form the bi- and trisyllabic sequences. In itself this seems obvious since the bi- and trisyllabic sequences are articulatorily much more difficult to pronounce than the monosyllabic sequences $[6,38]$. The importance, however, is that this establishes that MRR for the monosyllabic sequences and MRR for the bi- and trisyllabic sequences should be separate outcome measures that should both be included in the MRR task report. Furthermore, we included in the protocol that the bi- and trisyllabic sequences should not be administered if children could not produce the monosyllabic sequences to reduce the burden of the test battery.

According to the assessment protocol, the test administrator is instructed to ask children to redo the sequence 
Table 10. Mean (and SD) syllable rate for each of the different sequence lengths per number of syllables

\begin{tabular}{|c|c|c|c|c|c|c|c|c|c|c|c|c|c|c|c|c|c|c|}
\hline \multirow[t]{2}{*}{$\begin{array}{l}\text { Syllables, } \\
n\end{array}$} & \multicolumn{3}{|c|}{$\begin{array}{l}/ \mathrm{pa} / \\
(n=992)\end{array}$} & \multicolumn{3}{|c|}{$\begin{array}{l}/ \mathrm{ta} / \\
(n=996)\end{array}$} & \multicolumn{3}{|c|}{$\begin{array}{l}/ \mathrm{ka} / \\
(n=990)\end{array}$} & \multicolumn{3}{|c|}{$\begin{array}{l}\text { /pataka/ } \\
(n=902)\end{array}$} & \multicolumn{3}{|c|}{$\begin{array}{l}\text { /pata/ } \\
(n=962)\end{array}$} & \multicolumn{3}{|c|}{$\begin{array}{l}\text { /taka/ } \\
(n=943)\end{array}$} \\
\hline & $n$ & $\mathrm{M}$ & SD & $n$ & $\mathrm{M}$ & SD & $n$ & $\mathrm{M}$ & $\mathrm{SD}$ & $n$ & $\mathrm{M}$ & SD & $n$ & $\mathrm{M}$ & SD & $n$ & $\mathrm{M}$ & $\mathrm{SD}$ \\
\hline 3 & 183 & 4.5 & 0.8 & 165 & 4.4 & 0.8 & 140 & 4.1 & 0.7 & 120 & 4.2 & 1.2 & 115 & 4.2 & 0.8 & 111 & 4.2 & 0.9 \\
\hline 4 & 158 & 4.6 & 0.7 & 161 & 4.4 & 0.7 & 143 & 4.2 & 0.7 & 142 & 4.1 & 0.9 & 156 & 4.5 & 1.0 & 119 & 4.5 & 0.9 \\
\hline 5 & 155 & 4.6 & 0.6 & 129 & 4.6 & 0.7 & 124 & 4.2 & 0.6 & 113 & 4.1 & 1.0 & 128 & 4.4 & 0.9 & 100 & 4.4 & 0.9 \\
\hline 8 & 75 & 4.6 & 0.5 & 76 & 4.4 & 0.6 & 90 & 4.2 & 0.6 & 63 & 3.9 & 0.8 & 92 & 4.5 & 0.9 & 83 & 4.3 & 0.7 \\
\hline 9 & 68 & 4.7 & 0.7 & 82 & 4.5 & 0.5 & 74 & 4.3 & 0.5 & 68 & 4.1 & 0.8 & 60 & 4.6 & 0.7 & 70 & 4.4 & 0.7 \\
\hline 10 & 43 & 4.6 & 0.6 & 61 & 4.5 & 0.5 & 45 & 4.2 & 0.5 & 62 & 3.9 & 0.8 & 53 & 4.6 & 0.7 & 65 & 4.5 & 0.7 \\
\hline$>10$ & 91 & 4.7 & 0.5 & 111 & 4.6 & 0.6 & 108 & 4.3 & 0.6 & 120 & 3.9 & 0.8 & 135 & 4.5 & 0.7 & 148 & 4.5 & 0.7 \\
\hline
\end{tabular}

up to 2 times again if he/she suspects it was not performed at the child's maximum capacity. In $14 \%$ of the cases, the child was asked to repeat a sequence, and in $2 \%$ of the cases, the child got a third attempt of one or more of the sequences. Our results showed that most children were actually the fastest at the first attempt compared to the other attempts; only about $20 \%$ of the children were faster on the second or third attempt. However, it seems important to give children a second or even a third attempt if the administrator expect children to be even faster, because for about $12 \%$ of the attempts, the child was faster at the second or third attempt. In most protocols, there is a gradual build-up of number of syllables and pace of the sequences to be produced. After several trials, the children can be asked to produce the sequence as quickly as possible without an example. The expectation was that children show the fastest rate with the instruction to go as fast as possible, but this has not been explored in any published data. On the surface, a substantial number of children performed the fastest MRR with the instruction to go faster. The results showed a distinct pattern underneath. For the 2 monosyllabic sequences / pa/ and / ta/ and for the bi- and trisyllabic sequences, the instruction to perform the sequence "as fast as possible" yielded the fastest MRR, while for $/ \mathrm{ka} /$, the performance was the same between the 2 instructions. However, the effect sizes are very small, and therefore, it is debatable if the difference between the performance for the 2 instructions is clinically relevant. The difference could be an effect of learning how to conduct the task. Within the protocol, the child first practices the sequence (build up), and when the child is familiar with the sequence, the child is asked to produce it as fast as possible, thereby requiring maximal performance. However, we noticed children going louder and not that much faster, and the effect size of the difference between the 2 instructions is very small. The advice is to choose the fastest attempt that can be performed with either the last or the second last instruction and/or attempt.

Recent studies report the use of $10-12$ syllables $[9,14-$ 16]. However, this study showed that a large number of especially the youngest children do not reach the criterion of sequence length 10 . Instead, they produce sequence lengths in the range from 3 to about 10 syllables after exclusion of the first and last syllables. Gadesmann and Miller [21] compared the following methods of the same sequence children pronounced: number of syllables for the first $5 \mathrm{~s}$, the time of pronouncing a number of repetitions (5 times) and the total duration of the maximum sequence length uttered in one breath, and thereby showed that the results are identical irrespective of the method of assessment. Based on this study and our own data, we conclude that a sequence of at least 5 syllables, such that the mean rate is based on measuring the duration of at least 3 syllables, is sufficient.

MRR is the most common measure, but in the literature, there are also indications that other measures of the MRR task can provide valuable information on the development of speech motor skills and therefore a better understanding of the underlying problems in children with MSD. In children with MSD, measuring speech variability can yield important information about the speech motor control system and to support the identification, assessment, and treatment of the underlying speech process $[12,15,25,38-41]$. The coefficient of variation of the syllable durations could be added to investigate the variability of the sequences, as well as the normalized pairwise variability index, which in previous studies has been used to investigate stress-timing and syllable-timing [42, 43]. 
Table 11. Intraclass correlation coefficients for the comparison of syllable rate for each of the sequence lengths 3-9 with 10 or more

\begin{tabular}{|c|c|c|c|c|c|}
\hline Sequence & $\begin{array}{l}\text { Comparison } \\
\text { syllables }\end{array}$ & $n$ & ICC & $\mathrm{CI} \mathrm{lb}$ & CI ub \\
\hline \multirow[t]{7}{*}{ /pa/ } & 3 with 10 & 92 & 0.904 & 0.858 & 0.936 \\
\hline & 4 with 10 & 92 & 0.945 & 0.918 & 0.963 \\
\hline & 5 with 10 & 92 & 0.966 & 0.949 & 0.977 \\
\hline & 6 with 10 & 92 & 0.975 & 0.962 & 0.983 \\
\hline & 7 with 10 & 92 & 0.985 & 0.978 & 0.990 \\
\hline & 8 with 10 & 92 & 0.994 & 0.991 & 0.996 \\
\hline & 9 with 10 & 92 & 0.997 & 0.996 & 0.998 \\
\hline \multirow[t]{7}{*}{$/ \mathrm{ta} /$} & 3 with 10 & 105 & 0.882 & 0.831 & 0.918 \\
\hline & 4 with 10 & 105 & 0.910 & 0.871 & 0.938 \\
\hline & 5 with 10 & 105 & 0.936 & 0.907 & 0.956 \\
\hline & 6 with 10 & 105 & 0.965 & 0.949 & 0.976 \\
\hline & 7 with 10 & 105 & 0.978 & 0.968 & 0.985 \\
\hline & 8 with 10 & 105 & 0.991 & 0.987 & 0.994 \\
\hline & 9 with 10 & 105 & 0.995 & 0.993 & 0.997 \\
\hline \multirow[t]{7}{*}{$/ \mathrm{ka} /$} & 3 with 10 & 102 & 0.843 & 0.776 & 0.891 \\
\hline & 4 with 10 & 102 & 0.904 & 0.886 & 0.934 \\
\hline & 5 with 10 & 102 & 0.929 & 0.897 & 0.952 \\
\hline & 6 with 10 & 102 & 0.957 & 0.936 & 0.970 \\
\hline & 7 with 10 & 102 & 0.977 & 0.966 & 0.984 \\
\hline & 8 with 10 & 102 & 0.986 & 0.980 & 0.991 \\
\hline & 9 with 10 & 102 & 0.995 & 0.993 & 0.997 \\
\hline \multirow[t]{7}{*}{ /pataka/ } & 3 with 10 & 58 & 0.881 & 0.807 & 0.928 \\
\hline & 4 with 10 & 58 & 0.899 & 0.835 & 0.939 \\
\hline & 5 with 10 & 58 & 0.913 & 0.857 & 0.947 \\
\hline & 6 with 10 & 58 & 0.962 & 0.936 & 0.977 \\
\hline & 7 with 10 & 58 & 0.982 & 0.969 & 0.989 \\
\hline & 8 with 10 & 58 & 0.983 & 0.972 & 0.990 \\
\hline & 9 with 10 & 58 & 0.989 & 0.981 & 0.993 \\
\hline \multirow[t]{7}{*}{ /pata/ } & 3 with 10 & 86 & 0.735 & 0.620 & 0.819 \\
\hline & 4 with 10 & 86 & 0.806 & 0.718 & 0.869 \\
\hline & 5 with 10 & 86 & 0.869 & 0.806 & 0.913 \\
\hline & 6 with 10 & 86 & 0.918 & 0.877 & 0.946 \\
\hline & 7 with 10 & 86 & 0.953 & 0.929 & 0.969 \\
\hline & 8 with 10 & 86 & 0.978 & 0.966 & 0.985 \\
\hline & 9 with 10 & 86 & 0.992 & 0.988 & 0.995 \\
\hline \multirow[t]{7}{*}{ /taka/ } & 3 with 10 & 105 & 0.824 & 0.752 & 0.877 \\
\hline & 4 with 10 & 105 & 0.863 & 0.805 & 0.905 \\
\hline & 5 with 10 & 105 & 0.905 & 0.864 & 0.935 \\
\hline & 6 with 10 & 105 & 0.945 & 0.921 & 0.963 \\
\hline & 7 with 10 & 105 & 0.974 & 0.962 & 0.982 \\
\hline & 8 with 10 & 105 & 0.987 & 0.980 & 0.991 \\
\hline & 9 with 10 & 105 & 0.995 & 0.992 & 0.996 \\
\hline
\end{tabular}

ICC, intraclass correlation coefficient; CI lb, confidence interval lower bound; CI ub, confidence interval upper bound.
However, some reservation is required in this respect as the current speech-to-result set-up for most variability measures is not yet sufficiently automated to serve as an easily applicable analysis tool in the daily practice of speech therapists [42]. The goal for us is to see if and how the assessment of variability as an outcome measure of the MRR task could be implemented in the CAI.

In this study, we asked parents or caregivers whether their child had a history of hearing problems and if they had any doubts about his/her hearing. It is possible that the child could have a mild hearing problem because parents and caregivers can overlook a mild hearing problem [44]. In the Netherlands, the hearing of all children is recorded during the regular governmental hearing screening after 2 weeks after birth (neonatal screening) and at the age of 4 [45]. Furthermore, the research assistants were asked to pay particular attention to signs of hearing problems. This is why we did not include a whole hearing screening, but it is possible that a few children had a mild hearing problem.

In the field of adult MSD, there has been debate about the potential utility of nonspeech oral motor tasks [4649], and recently, Staiger et al. [50] suggested that MRR is not a speech-like skill, and therefore, MRR is unusable in clinical assessment of MSD in adults. We would like to stress here, however, that results that hold for adults with acquired disorders do not necessarily hold for children with developmental disorders. As pointed out elsewhere in this special issue following Bishop [51] and Karmiloff-Smith [52, 53], developmental disorders are characterized by association rather than dissociation of functions $[54,55]$. Whereas the adult speech production system is highly redundant, and the different processes and representations are highly overlearned, children have an incomplete system that is still in development. At the age of 4-6 years, children still make speech errors in conversational speech or in naming pictures that can be based on an incomplete phonological system or an immature motoric speech system [30]. The dissociation between MRR and other speech tasks found for adults thus cannot be extended to children. In fact, correlations between performance on speech tasks and different nonspeech motor tasks have been found in several groups of children with speech disorder, among which children with CAS [56] and children with Fetal Alcohol Spectrum Disorders [57].

In addition, and even more importantly, the MRR serves an important function in differential diagnosis of developmental speech disorders, as, for example, also expressed in the 2011 Speech-language pathology med- 
ical review guidelines from the American Speech-Language-Hearing Association [58]. Several studies have reported differences between children with and without MSD on the MRR [14, 15], and the MRR has been shown to be discriminative between CAS and developmental dysarthria $[14,25]$. We therefore propose that for a comprehensive speech assessment the following tasks should be administered: PN, NWI, WR, NWR, and MRR [32]. This study yields directions for administering the MRR tasks and norm values to interpret the performances relative to typically developing children. Research with diverse groups of children with SSD with the comprehensive test battery is required to validate the MRR and evaluate its contribution to the speech diagnosis. Such studies are currently conducted by our research group.

In summary, the new MRR protocol describes how to assess children from 3 years of age; if a child cannot perform $>2$ monosyllabic sequences, the session can be ended. In the clinical report of the MRR, the score for the monosyllabic and for the bi- and trisyllabic sequences must be given separately. Children do not have to be encouraged to perform a sequence of at least 10 syllables. For each MRR sequence, the test administrator should analyze the attempts the child has produced upon the last 2 instructions and then determine which attempt was the fastest.

\section{Acknowledgment}

The authors would like to thank all parents, children, the SLP students, and SLPs for their participations.

\section{Statement of Ethics}

Parents and guardians have given their written informed consent, and the schools/daycares have given their consent to recruit the children at their facility.

The research institute's committee on human research (Radboudumc) declared the study can be carried out (in the Netherlands) without an approval by an accredited research ethics committee.

\section{Disclosure Statement}

The authors have no conflicts of interest to declare.

\section{Funding Sources}

No funding was given.

\section{Author Contributions}

All the authors fulfil the ICMJE Criteria for Authorship.

\section{References}

1 Shriberg LD, Strand EA, Fourakis M, Jakielski KJ, Hall SD, Karlsson HB, et al. A diagnostic marker to discriminate childhood apraxia of speech from speech delay: I. Development and description of the pause marker. J Speech Lang Hear Res. 2017 Apr; 60(4):S1096-117.

2 Dodd B, McIntosh B. The input processing, cognitive linguistic and oro-motor skills of children with speech difficulty. Int J Speech Lang Pathol. 2008;10(3):169-78.

3 Rvachew S, Hodge M, Ohberg A. Obtaining and interpreting maximum performance tasks from children: A tutorial. J Speech Lang Pathol Audiol. 2005;29:146.

4 Shriberg LD. Diagnostic markers for child speech-sound disorders: introductory comments. Clin Linguist Phon. 2003 Oct-Nov; 17(7):501-5.

5 Shriberg LD, Strand EA, Fourakis M, Jakielski KJ, Hall SD, Karlsson HB, et al. A Diagnostic Marker to Discriminate Childhood Apraxia of Speech From Speech Delay: IV. The Pause Marker Index. J Speech Lang Hear Res. 2017 Apr;60(4):S1153-69.
6 Wong AW, Allegro J, Tirado Y, Chadha N, Campisi P. Objective measurement of motor speech characteristics in the healthy pediatric population. Int J Pediatr Otorhinolaryngol. 2011 Dec;75(12):1604-11.

7 McCauley RJ, Strand EA. A review of standardized tests of nonverbal oral and speech motor performance in children. Am J Speech Lang Pathol. 2008 Feb;17(1):81-91.

8 Icht M, Ben-David BM. Oral-diadochokinesis rates across languages: english and Hebrew norms. J Commun Disord. 2014 Mar-Apr;48: 27-37.

9 Rvachew S, Brosseau-Lapré F. Developmental phonological disorders: Foundations of clinical practice. Plural Pub.; 2012.

10 Wang YT, Kent RD, Duffy JR, Thomas JE. Analysis of diadochokinesis in ataxic dysarthria using the motor speech profile program. Folia Phoniatr Logop. 2009;61(1):1-11.

11 Yang CC, Chung YM, Chi LY, Chen HH, Wang YT. Analysis of verbal diadochokinesis in normal speech using the diadochokinetic rate analysis program. J Dent Sci. 2011;6(4): 221-6.
12 Williams P, Stackhouse J. Rate, accuracy and consistency: diadochokinetic performance of young, normally developing children. Clin Ling \& Phonet. 2000;14(4):267-93.

13 Kent RD, Kent JF, Rosenbek JC. Maximum performance tests of speech production. J Speech Hear Disord. 1987 Nov;52(4):367-87.

14 Thoonen G, Maassen B, Wit J, Gabreels F, Schreuder R. The integrated use of maximum performance tasks in differential diagnostic evaluations among children with motor speech disorders. Clin Linguist Phon. 1996; 10(4):311-36.

15 Wit J, Maassen B, Gabreëls FJ, Thoonen G. Maximum performance tests in children with developmental spastic dysarthria. J Speech Hear Res. 1993 Jun;36(3):452-9.

16 Yaruss JS, Logan KJ. Evaluating rate, accura$\mathrm{cy}$, and fluency of young children's diadochokinetic productions: a preliminary investigation. J Fluency Disord. 2002;27(1):65-85.

17 Juste FS, Rondon S, Sassi FC, Ritto AP, Colalto CA, Andrade CR. Acoustic analyses of diadochokinesis in fluent and stuttering children. Clinics (São Paulo). 2012;67(5):409-14. 
18 Vance M, Stackhouse J, Wells B. Speech-production skills in children aged 3-7 years. Int $J$ Lang Commun Disord. 2005 Jan-Mar;40(1): 29-48.

19 Padovani M, Gielow I, Behlau M. Phonarticulatory diadochokinesis in young and elderly individuals. Arq Neuropsiquiatr. 2009 Mar; 67(1):58-61.

20 Shriberg LD, Lohmeier HL, Campbell TF, Dollaghan CA, Green JR, Moore CA. A nonword repetition task for speakers with misarticulations: the Syllable Repetition Task (SRT). J Speech Lang Hear Res. 2009 Oct; 52(5):1189-212.

21 Gadesmann M, Miller N. Reliability of speech diadochokinetic test measurement. Int J Lang Commun Disord. 2008 Jan-Feb;43(1):41-54.

22 Milenkovic P. Time-frequency analysis for 32-bit windows. Madison (WI): University of Wisconsin; 2001.

23 Hodge MM, Gotzke CL. Minimal pair distinctions and intelligibility in preschool children with and without speech sound disorders. Clin Linguist Phon. 2011 Oct;25(10): 853-63.

24 Boersma P, Weenink D. Praat: doing phonetics by computer (Version 6.0.21). 2016.

25 Thoonen G, Maassen B, Gabreels F, Schreuder R; G. THOONEN B. MAASSEN F. GABREELS R. Validity of maximum performance tasks to diagnose motor speech disorders in children. Clin Linguist Phon. 1999; 13(1):1-23.

26 Lewis BA, Freebairn LA, Hansen AJ, Iyengar SK, Taylor HG. School-age follow-up of children with childhood apraxia of speech. Lang Speech Hear Serv Sch. 2004 Apr;35(2):12240.

27 Murray E, McCabe P, Heard R, Ballard KJ. Differential diagnosis of children with suspected childhood apraxia of speech. J Speech Lang Hear Res. 2015 Feb;58(1):43-60.

28 Ozanne A. Childhood apraxia of speech. Differential diagnosis and treatment of children with speech disorder. 2005, pp. 71-82.

29 Bradford A, Dodd B. Do all speech-disordered children have motor deficits? Clin Linguist Phon. 1996;10(2):77-101.

30 Maassen B, Terband H. Process-oriented diagnosis of childhood and adult apraxia of speech (CAS and AOS). In: Redford MA, editor. The handbook of speech production. 2015, pp. 331-350.

31 Maassen B, van Haaften L, Diepeveen S, Terband H, van den Engel-Hoek L, Veenker T, et al. Computer Articulatie Instrument. Amsterdam. Boom Uitgevers. 2019.

32 Van Haaften L, Diepeveen S, van den EngelHoek L, de Swart B, Maassen B. The develop- ment and clinimetric evaluation of a speech production test for children: Reliability and validity of the Computer Articulation Instrument (CAI). In: Maassen B, van Lieshout $P$, Terband H, editors. Stem-, Spraak-en Taalpathologie; 6th International Conference on Speech Motor Control Groningen: Abstracts. Groningen; 2017, vol 17.

33 Modolo DJ, Berretin-Felix G, Genaro KF, Brasolotto AG. Oral and vocal fold diadochokinesis in children. Folia Phoniatr Logop. 2011;63(1):1-8

34 Ackermann H, Hertrich I, Hehr T. Oral diadochokinesis in neurological dysarthrias. Folia Phoniatr Logop. 1995;47(1):15-23.

35 van Haaften L, Diepeveen S, van den EngelHoek L, Jonker M, de Swart B, Maassen B. The Psychometric Evaluation of a Speech Production Test Battery for Children: The Reliability and Validity of the Computer Articulation Instrument. J Speech Lang Hear Res. Forthcoming.

36 Henry CE. The development of oral diadochokinesia and non-linguistic rhythmic skills in normal and speech-disordered young children. Clin Linguist Phon. 1990;4(2):121-37.

37 Tiffany WR. The effects of syllable structure on diadochokinetic and reading rates. J Speech Hear Res. 1980 Dec;23(4):894-908.

38 Terband H, Maassen B, van Lieshout P, Nijland L. Stability and composition of functional synergies for speech movements in children with developmental speech disorders. J Commun Disord. 2011 Jan-Feb;44(1):59-74.

39 McCabe P, Rosenthal JB, McLeod S. Features of developmental dyspraxia in the general speech-impaired population? Clin Linguist Phon. 1998;12(2):105-26.

40 Murdoch BE, Attard MD, Ozanne AE, Stokes $\mathrm{PD}$. Impaired tongue strength and endurance in developmental verbal dyspraxia: a physiological analysis. Eur J Disord Commun. 1995; 30(1):51-64.

41 Preston JL, Koenig LL. Phonetic variability in residual speech sound disorders: exploration of subtypes. Top Lang Disord. 2011 Apr; 31(2):168-84.

42 van Brenk F. Objectieve beoordeling van spraakproblemen bij sprekers met dysartrie. Stem. Spraak-en Taalpathologie; 2015. p. 20.

43 Grabe E, Low EL. Durational variability in speech and the rhythm class hypothesis. Papers Laborat Phonol; 2002. p. 7.

44 Lo PS, Tong MC, Wong EM, van Hasselt CA. Parental suspicion of hearing loss in children with otitis media with effusion. Eur J Pediatr. 2006 Dec;165(12):851-7.

45 Lanting C, Deurloo J, Wiefferink C, Uilenburg N. JGZ-richtlijn Vroegtijdige opsporing van gehoorverlies bij kinderen en jongeren (0 tot 18 jaar). JGZ Tijdschrift voor jeugdgezondheidszorg 2017;49:77-80.

46 Kent RD. Nonspeech oral movements and oral motor disorders: A narrative review. Am J Speech Lang Pathol. 2015 Nov;24(4):76389.

47 Maas E. Speech and nonspeech: what are we talking about? Int J Speech Lang Pathol. 2017 Aug;19(4):345-59.

48 Weismer G. Philosophy of research in motor speech disorders. Clin Linguist Phon. 2006 Jul;20(5):315-49.

49 Ziegler W, Ackermann H. Neuromotor speech impairment: it's all in the talking. Folia Phoniatr Logop. 2013;65(2):55-67.

50 Staiger A, Schölderle T, Brendel B, Bötzel K, Ziegler W. Oral motor abilities are task dependent: A factor analytic approach to performance rate. J Mot Behav. 2017 Sep-Oct;49(5): 482-93.

51 Bishop DV. Cognitive neuropsychology and developmental disorders: uncomfortable bedfellows. Q J Exp Psychol A. 1997 Nov; 50(4):899-923.

52 Karmiloff-Smith A. The tortuous route from genes to behavior: A neuroconstructivist approach. Cogn Affect Behav Neurosci. 2006 Mar;6(1):9-17.

53 Karmiloff-Smith A, Scerif G, Ansari D. Double dissociations in developmental disorders? Theoretically misconceived, empirically dubious. Cortex. 2003 Feb;39(1):161-3.

54 Terband H, Maassen B, Maas E. Toward a model of pediatric speech sound disorders (SSD) for differential diagnosis and therapy planning. Speech Motor Control in normal and disordered speech: Future developments in theory and methodology. P. Van Lieshout, B. Maassen and H. Terband. Rockville (MD): American Speech-Language-Hearing Association; 2016

55 Terband H, Maassen B, Maas E. A psycholinguistic framework for diagnosis and treatment planning of developmental speech disorders. Folia Phoniatr Logop. Forthcoming.

56 Nijland L, Terband H, Maassen B. Cognitive Functions in Childhood Apraxia of Speech. J Speech Lang Hear Res. 2015 Jun;58(3):55065.

57 Terband H, Spruit M, Maassen B. Speech Impairment in Boys With Fetal Alcohol Spectrum Disorders. Am J Speech Lang Pathol. 2018 Nov;27(4):1405-25.

58 ASHA. Speech-language pathology medical review guidelines: American1 Speech-Language-Hearing Association, 2011. Available from: www.asha.org/policy. 